\title{
A study of AGB stars in LMC clusters
}

\author{
Thomas Lebzelter ${ }^{1}$, Michael T. Lederer ${ }^{1}$, Sergio Cristallo $^{2}$, \\ Oscar Straniero ${ }^{2}$ and Kenneth H. Hinkle ${ }^{3}$ \\ ${ }^{1}$ Department of Astronomy, University of Vienna, Türkenschanzstraße 17, \\ A-1180 Vienna, Austria \\ email: lebzelter@astro.univie.ac.at \\ ${ }^{2}$ INAF, Teramo, Italy \\ email: straniero@oa-teramo.inaf.it \\ ${ }^{3}$ NOAO, Tucson, USA \\ email: hinkle@noao.edu
}

\begin{abstract}
LMC clusters offer an outstanding opportunity to investigate the late stages of stellar evolution of stars in the mass range between 1.5 and $2 \mathrm{M}_{\odot}$. In this presentation we will focus on our results on mixing events during the evolution along the Asymptotic Giant Branch (AGB). Surface abundances have been determined for a number of cluster AGB stars from high resolution near infrared spectra. We show for the first time the evolution of $\mathrm{C} / \mathrm{O}$ and ${ }^{12} \mathrm{C} /{ }^{13} \mathrm{C}$ ratios along a cluster AGB. The change of both quantities due to dredge up events is compared with model predictions. Our results indicate the late occurrence of a moderate extra-mixing in some cases.
\end{abstract}

Keywords. stars: abundances, stars: AGB and post-AGB, stars: evolution, galaxies: individual (LMC), Magellanic Clouds, galaxies: star clusters

\section{Introduction}

Stars of low and intermediate mass contribute significantly to the cosmic cycle of matter. The evolutionary phase during which they actually enrich the interstellar medium with freshly produced elements, however, is a quite short one, namely the Asymptotic Giant Branch (AGB) phase. The enrichment process itself can be roughly separated into three (of course connected) steps: the production of the elements in the interior, the dredge up of the material to the surface, and the mass loss (e.g., Busso et al. 1999)

In this paper the focus is set to the dredge up process. The dredge up events on the AGB are called the third dredge up, with the first dredge up occurring at the bottom of the RGB and the second dredge up acting in intermediate mass stars after the ignition of core He burning. Stellar evolution models (e.g., Herwig 2000; Straniero et al. 2006; Karakas \& Lattanzio 2007) agree qualitatively on the basic characteristics of the third dredge up, but details on onset and efficiency are still awaiting constraints from observational tests.

With our study of AGB stars in stellar clusters we want to contribute to our understanding of the mixing processes during the AGB phase. Stellar clusters, generally consisting of single stellar populations, provide the required sample of AGB stars homogeneous in mass and metallicity. The intermediate age clusters in the Magellanic Clouds provide samples of stars that allow to measure the change of surface abundances due to these mixing events. The probes we use in our study are the $\mathrm{C} / \mathrm{O}$ ratio and the carbon isotopic ratio ${ }^{12} \mathrm{C} /{ }^{13} \mathrm{C}$. Both quantities are affected by the dredge up of ${ }^{12} \mathrm{C}$ produced in the stellar interior. 


\section{Target selection}

Our project aims to obtain the above mentioned abundance ratios in a variety of clusters in the age range 1.4 to $1.9 \mathrm{Gyr}$ corresponding to turn-off masses between roughly 1.4 and $1.8 \mathrm{M}_{\odot}$. The lower mass limit is close to the typical model predicted minimum mass for third dredge up. The upper limit is given by the need for a sufficient number of stars currently on the AGB. Among the LMC clusters fulfilling this requirement we started our study with NGC 1846 and NGC 1978. In both clusters a number of AGB stars have been identified already before by Lloyd Evans (1983) and Frogel et al. (1990). Furthermore, luminous carbon stars were detected in both of them (Frogel et al. 1990) indicating that third dredge up occurred in these clusters.

Both clusters have a similar metallicity around $[\mathrm{Fe} / \mathrm{H}]=-0.4$ (Grocholski et al. 2006; Mucciarelli et al. 2007). The AGB stars of NGC 1846 have a mass of about $1.8 \mathrm{M}_{\odot}$ (Lebzelter \& Wood 2007), while the colour - magnitude diagram of NGC 1978 indicates a somewhat lower mass around 1.4-1.5 $\mathrm{M}_{\odot}$ (Mucciarelli et al. 2007). NGC 1978 is elongated, but no indication for a second population (resulting from a merging of two clusters) could be found (Mucciarelli et al. 2007). On the opposite Mackey \& Broby Nielsen (2007) claim the detection of two populations in NGC 1846 separated in age by about 300 Myr. Both clusters were found to be very homogeneous in metallicity.

\section{Observations and abundance determination}

Spectra of a large fraction of stars along the whole AGB were observed in both clusters. We used the Phoenix Spectrograph at Gemini South to obtain spectra around the CO 3-0 band head and strong $\mathrm{OH}$ lines in the H-band. In addition we observed the same stars also in a small region in the K-band including lines of ${ }^{12} \mathrm{CO}$ and ${ }^{13} \mathrm{CO}$.

Abundance and isotopic ratios were determined by fitting of the observations with synthetic spectra. These spectra are based on model atmospheres that were calculated with the COMARCS code, a modified version of the MARCS code. Starting values for $T_{\text {eff }}$ and $L$ were derived from broad-band, near-infrared photometry. The metallicity of the cluster was taken as fixed. While spectra of both oxygen and carbon rich stars were obtained, difficulties in modelling the C-stars lead to higher uncertainties for the abundances in these objects. A detailed description on data analysis, synthetic spectra calculation and abundance determination can be found in Lebzelter et al. (2008).

\section{Results}

\section{1. $N G C 1846$}

We present the results for the two clusters separately. More details on the findings for NGC 1846 have been published already in Lebzelter et al. (2008). For the O-rich stars in NGC 1846 we determined C/O ratios between 0.2 and 0.65 with an uncertainty from \pm 0.05 to \pm 0.1 dex. Carbon isotopic ratios between 12 and 60 were found for these stars. Both dredge up indicators show obvious variations from star to star, but nicely correlated with each other as expected.

Indeed the most luminous O-rich star in NGC 1846 has the highest $\mathrm{C} / \mathrm{O}$ and ${ }^{12} \mathrm{C} /{ }^{13} \mathrm{C}$ and for the least luminous star in our sample we found the lowest values. However, there is no simple relation in between. This may be explainable by the luminosity changes due to the thermal pulse cycle which can significantly affect a star's location in the colourmagnitude-diagram. It is also possible that NGC 1846 indeed harbours two populations and the observed AGB consists of a mixture of stars of different age. There may also 


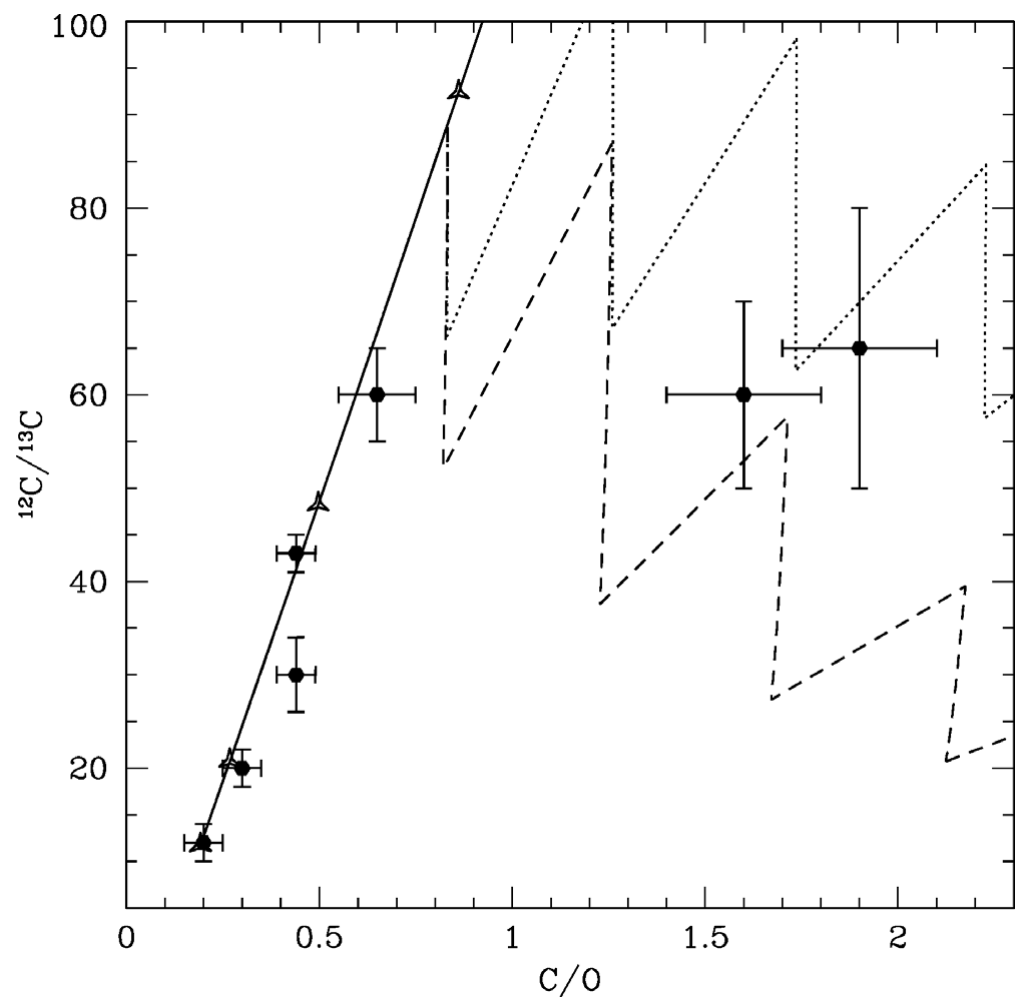

Figure 1. C/O versus ${ }^{12} \mathrm{C} /{ }^{13} \mathrm{C}$ for all the sampled stars. Predicted values from models with and without extra-mixing are shown. Filled circles with error bars are our measurements in NGC 1846. Solid line refers to our reference model, while the dotted and dashed ones refer to models with $T_{\mathrm{lim}}=35 \times 10^{6} \mathrm{~K}$ and $T_{\mathrm{lim}}=40 \times 10^{6} \mathrm{~K}$, respectively. The symbols along the solid model line mark the values predicted after each dredge up event.

be a correlation with the pulsation mode — see Lebzelter et al. (2008) for a detailed discussion.

Only for two C-stars we could derive a $\mathrm{C} / \mathrm{O}$ and ${ }^{12} \mathrm{C} /{ }^{13} \mathrm{C}$ ratio with reasonable error bars. While the $\mathrm{C} / \mathrm{O}$ ratio increases as expected to values around 1.8 , the isotopic ratio was found to stay close to 60 , the value found for the most luminous O-rich star.

We also made a comparison of our findings for that cluster with predictions from the FRANEC stellar evolution code (Chieffi et al. 1998). Figure 1 illustrates the outcome of this comparison. The most likely model based on the global cluster parameters is indicated by a solid line. The model predictions for the O-rich stars are nicely reproduced by the observations if we assume an oxygen overabundance of about +0.2 dex (leading to a $\mathrm{C} / \mathrm{O}$ ratio of 0.2 at the beginning of the $\mathrm{AGB}$ phase). On the other hand, the ${ }^{12} \mathrm{C} /{ }^{13} \mathrm{C}$ ratio found in the $\mathrm{C}$-stars cannot be explained with standard models for the third dredge up. The most obvious explanation for this observational pattern is the occurrence of a mixing process able to bridge the radiative gap between the cool bottom of the convective envelope and the hot H-burning zone. A similar process seems to explain some abundance anomalies in red giants stars (see, e.g., Charbonnel 1995). It is usually referred to as extra or deep mixing. The values found indicate that the envelope material of the C-stars could have been exposed to a temperature on the order of $30-40 \times 10^{6} \mathrm{~K}$. At that temperature and on an AGB time scale, ${ }^{12} \mathrm{C}$ is partially converted into ${ }^{13} \mathrm{C}$, but only marginally into 
${ }^{14} \mathrm{~N}$. The two dotted lines in Fig. 1 show the predicted values if extra-mixing is included (see Lebzelter et al. 2008 for details).

Beside the $\mathrm{C} / \mathrm{O}$ ratio we also made an attempt to measure the change of the fluorine abundance along the AGB. Abundances were derived from only one blended HF line, thus especially the absolute values have to be taken with some caution. A clear increase in the F abundance with luminosity was seen in the O-rich NGC 1846 AGB stars. Models predict also an increase in the $\mathrm{F}$ abundance, but less than observed.

\section{2. $N G C 1978$}

This cluster is an interesting candidate for comparison with our findings for NGC 1846 . While having a very similar metallicity it is clearly older and thus the stars on the AGB have a smaller mass. As mentioned above, this mass is close to the predicted minimum mass for the occurrence of third dredge up.

Five oxygen rich AGB stars were analysed in this cluster. Opposite to NGC 1846 they all show very similar values of $\mathrm{C} / \mathrm{O}$ and ${ }^{12} \mathrm{C} /{ }^{13} \mathrm{C}$ close to 0.2 and 12 , respectively. Thus we see no indication for an ongoing dredge up in these stars, although they cover some range in luminosity. Again, only $2 \mathrm{C}$-stars could be analysed giving $\mathrm{C} / \mathrm{O}$ ratios of 1.3 and 1.35, respectively. Also different from NGC 1846, the C-stars in that cluster show very high values of the carbon isotopic ratio close to the value predicted by the standard model. According to these preliminary results no extra-mixing has to be included to reproduce the observations in this cluster. Compared with the findings for field stars (Smith \& Lambert 1990) the isotopic ratio measured in these objects is untypically high. We note that it is even above the solar value (89).

In Fig. 2 the resulting abundance ratios for NGC 1978's AGB stars are plotted. The big gap between the O-rich stars and the C-stars is intriguing and at the moment lacks a clear explanation. We list here several possible reasons for this gap:

- The cluster may consist of two stellar populations with different age. The O-rich stars and the C-rich stars would then have different masses and ages. The existence of two populations has been suggested by Alcaino et al. (1999), but a more recent detailed study by Mucciarelli et al. (2007) could not confirm this.

- We may assume an abundance scatter, especially in the $\alpha$-elements, within the cluster. In that case the starting composition of the AGB stars would show some scatter which affected the dredge up efficiency.

- Models predict combinations of stellar parameters, where the dredge up is so efficient that the star turns from O-rich to C-rich chemistry during one dredge up event (e.g., Herwig et al. 2000). However, the predicted metallicity where such an event should occur is much lower than the value found for NGC 1978.

- As the derived mass of the AGB stars in this cluster is very close to the mass limit for third dredge up predicted by stellar evolution models the findings are possibly explained by the following scenario: the O-rich stars found at the bottom of the AGB have a slightly lower mass than the C-stars at the top of the AGB. The limit for third dredge up may be exactly between the mass of the C-rich and O-rich stars. Then the O-rich stars would have not enough mass to encounter a third dredge up. However, dredge up models predict a "mild" dredge up (not producing a C-star) at the lower mass end which is not observed.

At this point none of the scenarios gives a satisfying explanation for the observations. A more detailed comparison with models will be given in a forthcoming paper (Lederer et al. in preparation). 


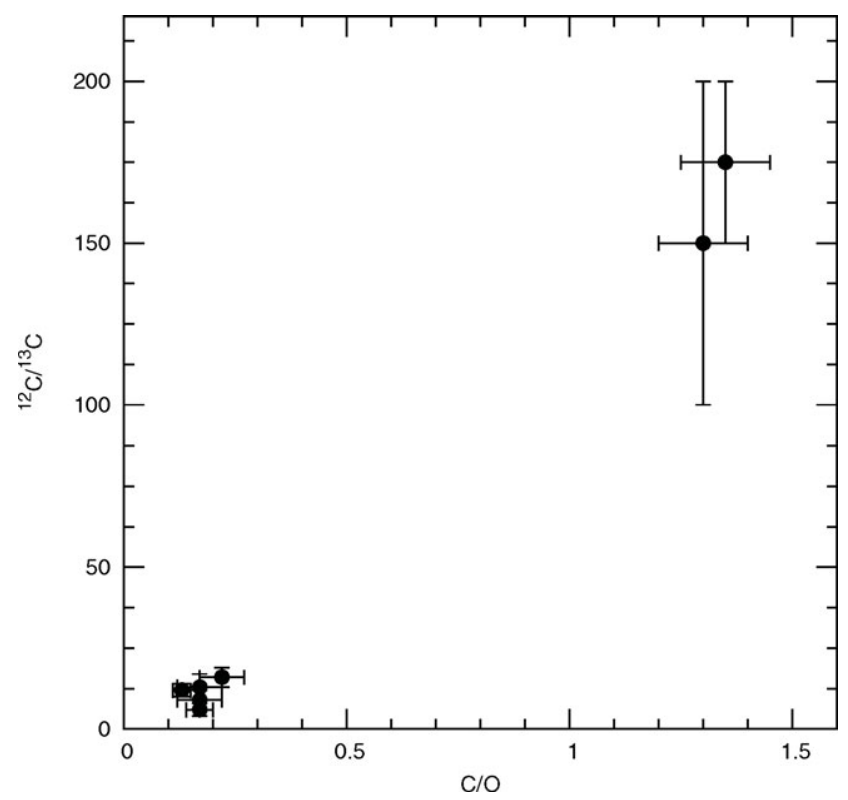

Figure 2. C/O versus ${ }^{12} \mathrm{C} /{ }^{13} \mathrm{C}$ for the sampled stars in NGC 1978. The lack of dredge up in the O-rich stars is obvious. The large error bars in the C-rich case result from both uncertainties in the determination of the stellar parameters and the insufficient accuracy of the line lists. For the other C-stars only a lower $\mathrm{C} / \mathrm{O}$ limit could be derived which is approximately 1.5.

\section{Conclusions}

Our observations clearly illustrate that AGB stars in globular clusters are well suited to test and constrain the description of the AGB phase in stellar evolution models. The need for an inclusion of extra-mixing has been demonstrated. The exact conditions for the occurrence of this mixing require further investigation. We will continue our study by extending the observational material towards other sets of stellar parameters and by intensifying comparison with stellar models. Finally, we emphasize the need for further improvements in the area of molecular line lists and dynamical model atmospheres to fully explore the wide variety of stellar clusters in the Magellanic Clouds.

\section{Acknowledgements}

This work was supported by the Austrian FWF under project number P18171-N02 and P20046-N16. MTL has been supported by the Austrian Academy of Sciences (DOC programme). Based on observations obtained at the Gemini Observatory. The spectra were obtained as part of program GS-2005B-C-7.

\section{References}

Alcaino, C., Liller, W., Alvarado, F., et al. 1999, A\& $A S, 135,103$

Busso, M., Gallino, R., \& Wasserburg, J. 1999, ARAA, 37, 239

Charbonnel, C. 1995, ApJ, 453, 41

Chieffi, A., Limongi, M., \& Straniero, O. 1998, ApJ, 502, 737

Frogel, J. A., Mould, J., \& Blanco, V. M., 1990, ApJ, 352, 96

Grocholski, A. J., Cole, A. A. Sarajedini, A., et al. 2006, AJ, 132, 1630

Herwig, F. 2000, $A \& A, 360,952$

Herwig, F., Blöcker, T., \& Driebe, T. 2000, MemSAI, 71, 745 
Karakas, A. \& Lattanzio, J. 2007, PASA, 24, 103

Lebzelter, T. \& Wood, P. R. 2007, A\&AA, 475, 643

Lebzelter, T., Lederer, M. T., Cristallo, S., et al. 2008, A\&SA, 486, 511

Lloyd Evans, T. 1983, MNRAS, 204, 985

Mackey, A. D. \& Broby Nielsen, P. 2007, MNRAS, 379, 151

Mucciarelli, A., Ferraro, F. R., Origlia, L., \& Fusi Pecci, F. 2007, AJ, 133, 2053

Smith, V. V. \& Lambert, D. L. 1990, ApJS, 72, 387

Straniero, O., Gallino, R., \& Cristallo, S. 2006, N.Phys.A, 777, 311

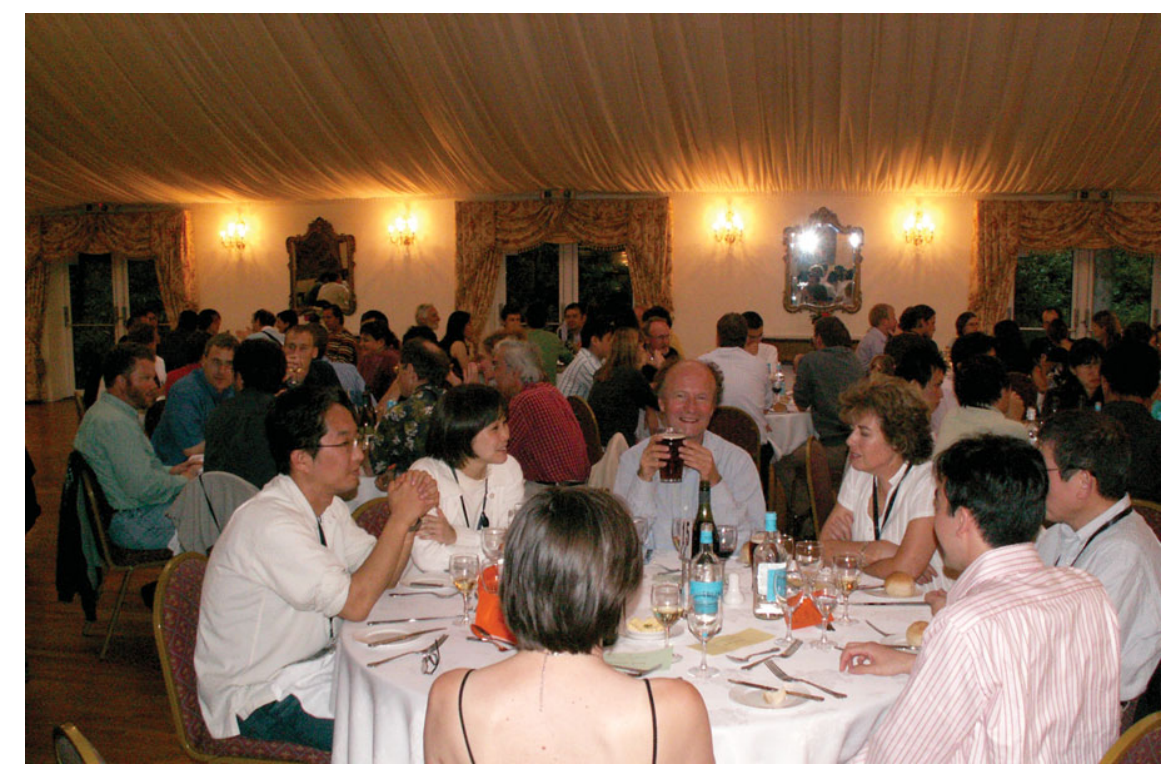

At the conference dinner inside Wrenbury Hall. 